\title{
Analysis on Information Sharing in Forest Product Supply Chain Based on Evolutionary Game Theory
}

\author{
Yi Tang ${ }^{1,2 *}$, Zhongwei Wang ${ }^{1}$, Yuan Zhang ${ }^{2}$, Ru Fang ${ }^{2}$ \\ ${ }^{1}$ School of Transportation and Logistics, Central South University of Forestry ang Technology, Changsha 411000, China \\ ${ }^{2}$ School of Public Management, Xiangtan University, Xiangtan 411105, China
}

Corresponding Author Email: tony@xtu.edu.cn

https://doi.org/10.18280/jesa.520610

Received: 18 May 2019

Accepted: 1 August 2019

\section{Keywords:}

forest product supply chain (FPSC), information sharing (IS), evolutionary game theory, equilibrium strategy

\begin{abstract}
This paper mainly explores the decision-making of enterprises on information sharing (IS) in forest product supply chain (FPSC). Firstly, several influencing factors of the IS between FPSC enterprises were identified according to the relevant literature, namely, the IS degree, information absorption capacity, the IS cost, incentive coefficient, penalty coefficient, risk coefficient and synergy coefficient. Based on these factors, the author constructed an evolutionary game model for the decision-making of the IS. Then, the dynamic evolution of the IS between FPSC enterprises was simulated on the Matlab, followed by the discussion on the impact of each factor on the IS decisions of each side. The results show that the IS between FPSC enterprises is promoted by the IS degree, information absorption capacity, incentive coefficient, penalty coefficient and synergy coefficient, but suppressed by the IS cost and risk coefficient. Finally, four countermeasures were suggested to enhance the IS between FPSC enterprises, including raising the IS awareness, enhancing information absorption capacity, creating an IS mechanism, and strengthening IS coordination. The research findings provide a reference for improving the IS between FPSC enterprises and enhancing the performance of the FPSC.
\end{abstract}

\section{INTRODUCTION}

Forest product supply chain (FPSC) is an ecological chain with ecological benefit as the core. For enterprises in the FPSC, information sharing (IS) offers an effective way to fully utilize information. However, FPSC enterprises are sometimes unenthusiastic to share information with each other, due to problems arising in the decision-making of the IS. For instance, the benefits of the IS might not offset the risk of secret leak or sharing cost. Therefore, it is very meaningful to explore the IS between FPSC enterprises.

The IS, essential to the information management of the FPSC, has been widely studied by scholars at home and abroad. For example, Lee et al. discussed show the IS affects the cost and operating performance of FPSC enterprises [1,2]. Dai et al. [3] probed into the causes of bullwhip effect, and the influence of the IS on this effect. Mishra et al. [4] analyzed the benefits of the 1-1 supply chain (SC) model, and found that the IS of demand reduces retailer profit in pull production. Some scholars constructed model based on the measuring criteria of conditional risk, and used the model to measure the risks before and after the IS of demand. Under the Bertrand competition between retailers, they examined the sensitivity of the profit of SC members to several influencing factors, namely, the variance in retailer cost, the substitutability of sold products, and cost correlation, and mathematically modelled the value of shared supply information [5-7]. Teunter et al. [8] confirmed that the information value is negative when demand follows a random walk and the retailer is slow to react. Zhao et al. [9] investigated how the IS of market demand between online retailers affects the SC's bullwhip effect and the supplier's inventory level. Babai et al. [10] discovered that the IS does not necessarily bring large benefits for the stock keep units (SKUs) with highly positive auto-correlated demand, and the IS benefits should be analyzed carefully for these units and the SKUs with less strong auto-correlated demand. Sabitha et al. [11] analyzed the value of the IS to a single-product serial SC with nonzero replenishment lead times, from the perspectives of reducing demand variance and mean inventory level. Rached et al. [12] studied the influence of the IS on a single-product serial SC, which consists of a supplier, a warehouse, a retailer and consumers, in the context of decentralized decision-making. Scott [13] evaluated the potential value of load offer sharing between the shipper and the carrier, and obtained the carrier's response before the scheduled pickup date. Under nonstationary stochastic demand and service-level constraints, Choudhary et al. [14] determined the value of vendor-managed inventory (VMI) in independent decision-making with the IS.

In the FPSC, the IS mechanism directly bears on the initiative and benefits of each enterprise. Feldmann et al. [15] assumed that the IS process involves opportunity behavior and incomplete and false information, and improved the Groves mechanism to incentive the provision of reliable and true information. In addition, the FPSC IS degree was evaluated from five aspects, showing that the upstream enterprises have a much higher IS degree than their downstream counterparts. This finding provides an important reference for implementing the IS incentives in the FPSC [16].

The evolutionary game theory originates from the concept of evolutionary stability strategy (ESS) proposed by ecologists Smith and Price. Contrary to traditional game theory, the 
evolutionary game theory holds the assumption of bounded rationality. According to Friedman, the evolutionary game theory is not only a theoretical knowledge, but also applicable to various real-world scenarios. Much research has been done on the application of this theory. Zhu et al. [17] investigates how to optimize the strategy of low carbon investment for suppliers and manufacturers in supply chains, and discuss the impacts of various factors on evolutionarily stable strategies. Additionally, we examine an incentive mechanism based on governmental subsidies to eliminate free riding and motivate co-investment. Furthermore, a case study and numerical examples are provided for illustration and simulation purposes, leading to several countermeasures and suggestions. After building a dynamic evolutionary game model, Zhang [18] studies the stability of the equilibrium in the game between the commercial banks and the closed-loop supply chain (CLSC) enterprises. By design of systematic mechanism based on system dynamics theory, capital chains of independent small and medium-sized enterprises (SMEs) on CLSC are organically linked together. Fang et al. [19] built up a multiagent evolutionary game model of renewable energy generation and transmission, and affirmed the necessity to enhance government regulation and its effectiveness. Zhang et al. [20] compared the dynamic game models of three financing patterns of photovoltaic (PV) enterprises. Zhao et al. [21] developed an evolutionary game model for electric power enterprises, analyzed the standard for renewable energy portfolio, explored the strategy of electric power enterprises for symbiotic evolution, and discussed the key parameters that affect the dynamic evolution of these enterprises. Qi et al. [22] proposed a noncooperative coordinated strategy of wind farm cluster based on game theory. Zhang and Li [23] constructed an evolutionary game model of cooperative haze control between governments on different levels, and used the model to analyze the dynamic evolution path of game system and the equilibrium strategy under no constraint, compensation mechanism, or penalty mechanism.

The IS between FPSC enterprises is not formed at one go, but through the continuous learning and adjustment of the enterprises. This process is a dynamic evolution process. However, most of the relevant studies have explored the IS in the FPSC based on the traditional game theory. This paper introduces the evolutionary game model to analyze the influencing factors of the IS between FPSC enterprises, with the aim to reduce the information uncertainty, improve operating efficiency, and enhance enterprise competitiveness of the FPSC. Based on the identified factors, the author put forward several countermeasures to improve the IS between FPSC enterprises.

\section{MODELLING}

\subsection{Hypotheses}

The following hypotheses were put forward to explore the IS between FPSC enterprises.

Hypothesis 1. The FPSC involves two types of enterprises, namely, the first-mover/proposer (A) and the secondmover/responder (B), each of which chooses between the IS and non-IS $\{$ IS, non-IS $\}$.

Hypothesis 2. The probabilities for A to choose the IS and the non-IS are $x$ and 1-x, respectively; the probabilities for $\mathrm{B}$ to choose the IS and the non-IS are $y$ and $1-y$, respectively; $x, y \in(0,1)$.

Hypothesis 3. Both incentive mechanism and penalty mechanism are introduced to encourage the IS between FPSC enterprises. The enterprises sharing the true and effective information will be incentivized, improving the benefit distribution. The enterprises taking speculative behavior will be penalized.

Hypothesis 4. The IS between A and B has a synergy effect, i.e. both sides will receive additional benefits. The non-IS between them brings no additional benefits.

\subsection{Variables}

According to the above hypothesis, the following variables were defined to facilitate the establishment of the evolutionary game model for the IS between FPSC enterprises.

(1) The IS degree $D_{i}(\mathrm{i}=A, B)$ : the authenticity and effectiveness of the information shared by an FPSC enterprise.

(2) Information absorption capacity $P_{i}(\mathrm{i}=A, B)$ : the mastery of the shared information of an FPSC enterprise. An enterprise with a high $P_{i}$ can effectively acquire, understand and utilize the information, and receive many additional benefits.

(3) The IS cost $C_{i}(\mathrm{i}=A, B)$ : the cost of human resources, equipment and information technology invested by each FPSC enterprise in the IS process.

(4) The incentive coefficient $\alpha$ : the incentive to the information sharing enterprises. The value of $\alpha$ is positively correlated with the IS degree. Here, the IS incentive is represented by $\alpha D_{i}$.

(5) The penalty coefficient $\beta$ : the penalty on the speculative FPSC enterprises. This coefficient guarantees the fairness of the FPSC.

(6) Risk coefficient $\gamma$ : the risk brought by the IS between FPSC enterprises. The value of $\gamma$ is positively correlated with the IS degree.

(7) Synergy coefficient $\mu$ : the synergy effect between information sharing enterprises. The value of $\mu$ is greater than 1 .

\subsection{Model establishment}

According to Hypothesis 1, the two types of enterprises involved in the evolutionary game only face two pure strategies: the IS and the non-IS. Through permutation and combination, the following four scenarios can be created:

Scenario 1: Both A and B choose the IS.

Under this scenario, the benefits that $\mathrm{A}$ and $\mathrm{B}$ acquire through the IS can be respectively described as:

$$
\begin{aligned}
& \mu \mathrm{D}_{b} \mathrm{P}_{\mathrm{a}}+\alpha \mathrm{D}_{\mathrm{a}}-\mathrm{C}_{\mathrm{a}}-\gamma \mathrm{D}_{\mathrm{a}} \\
& \mu \mathrm{D}_{a} \mathrm{P}_{\mathrm{b}}+\alpha \mathrm{D}_{\mathrm{b}}-\mathrm{C}_{\mathrm{b}}-\gamma \mathrm{D}_{\mathrm{b}}
\end{aligned}
$$

Scenario 2: Only A chooses the IS.

Under this scenario, no synergy effect occurs, because B chooses the non-IS. In addition, A bears the risk of the IS, while B benefits from the shared information, but with a penalty. Hence, under this scenario, the benefit of A through the IS and that of B through the non-IS can be respectively described as:

$$
\begin{aligned}
& -\mathrm{C}_{\mathrm{a}}-\gamma \mathrm{D}_{\mathrm{a}} \\
& \mathrm{D}_{a} \mathrm{P}_{\mathrm{b}}-\beta \mathrm{D}_{\mathrm{a}}
\end{aligned}
$$


Scenario 3: Only B chooses the IS.

Under this scenario, no synergy effect occurs, because A chooses the non-IS. In addition, B bears the risk of the IS, while A benefits from the shared information, but with a penalty. Hence, under this scenario, the benefit of A through the IS and that of B through the non-IS can be respectively described as:

$$
\mathrm{D}_{b} \mathrm{P}_{\mathrm{a}}-\beta \mathrm{D}_{\mathrm{b}}
$$

$$
-\mathrm{C}_{\mathrm{b}}-\gamma \mathrm{D}_{\mathrm{b}}
$$

Scenario 4: Both A and B choose the non-IS.

Under this scenario, neither side receive additional benefit. Thus, the benefits of A and B acquired through the non-IS are both 0 .

Combined with the previous hypotheses, the benefit matrix of the two types of enterprises can be established as Table 1.

Table 1. The benefit matrix of A and B

\begin{tabular}{c|c|c}
\hline $\mathrm{B}$ & IS $(y)$ & Non-IS $(1-y)$ \\
\hline $\mathrm{A}$ & $\left\{\mu D_{b} P_{a}+\alpha D_{a}-C_{a}-\gamma D_{a}, \mu D_{a} P_{b}+\alpha D_{b}-C_{b}-\gamma D_{b}\right\}$ & $\left\{-C_{a}-\gamma D_{a}, D_{a} P_{b}-\beta D_{a}\right\}$ \\
\hline IS $(x)$ & $\left\{D_{b} P_{a}-\beta D_{b},-C_{b}-\gamma D_{b}\right\}$ & $\{0,0\}$ \\
\hline Non-IS $(1-x)$ & &
\end{tabular}

\subsection{Reflector equation}

According to the benefit matrix and the evolutionary game theory, the expected benefit of A after choosing the IS and the non-IS can be respectively expressed as:

$$
\begin{gathered}
E_{a 1}=y\left(\mu \mathrm{D}_{b} \mathrm{P}_{\mathrm{a}}+\alpha \mathrm{D}_{\mathrm{a}}-\mathrm{C}_{\mathrm{a}}-\gamma \mathrm{D}_{\mathrm{a}}\right)+(1-y)\left(-\mathrm{C}_{\mathrm{a}}-\gamma \mathrm{D}_{\mathrm{a}}\right) \\
E_{a 2}=y\left(\mathrm{D}_{b} \mathrm{P}_{\mathrm{a}}-\beta \mathrm{D}_{\mathrm{b}}\right)+(1-y) \cdot 0
\end{gathered}
$$

Then, the mean expected benefit of A in the IS strategy and the non-IS strategy can be computed by:

$$
\overline{E_{a}}=x \cdot E_{a 1}+(1-x) \cdot E_{a 2}
$$

Thus, the replicator equation of $\mathrm{A}$ in the decision-making process can be established as:

$$
\begin{gathered}
F(x)=d x / d t=x \cdot\left(E_{a 1}-\overline{E_{a}}\right)= \\
x(1-x)\left[y\left(\mu D_{b} P_{a}+\alpha D_{a}-D_{b} P_{a}+\beta D_{b}\right)-C_{a}-\gamma D_{a}\right]
\end{gathered}
$$

Similarly, the expected benefit of B after choosing the IS and the non-IS can be respectively expressed as:

$$
\begin{gathered}
E_{b 1}=x\left(\mu D_{a} P_{b}+\alpha D_{b}-C_{b}-\gamma D_{b}\right)+(1-x)\left(-C_{b}-\gamma D_{b}\right) \\
E_{b 2}=x\left(D_{a} P_{b}-\beta D_{a}\right)+(1-x) \cdot 0
\end{gathered}
$$

Then, the mean expected benefit of B in the IS strategy and the non-IS strategy can be computed by:

$$
\overline{E_{b}}=y \cdot E_{b 1}+(1-y) \cdot E_{b 2}
$$

Thus, the replicator equation of $\mathrm{B}$ in the decision-making process can be established as:

$$
\begin{gathered}
F(y)=d y / d t=y \cdot\left(E_{b 1}-E_{b 2}\right)= \\
y(1-y)\left[x\left(\mu D_{a} P_{b}+\alpha D_{b}-D_{a} P_{b}+\beta D_{a}\right)-C_{b}-\gamma D_{b}\right]
\end{gathered}
$$

\section{MODEL ANALYSIS}

\subsection{Evolutionary equilibrium strategy}

The replicator equation of a party can achieve the equilibrium under the following conditions:

(1) If $F(x)=0$, the equilibrium of all replicator equations $x^{*}$ can be solved, i.e. $F\left(x^{*}\right)=0$.

(2) To ensure the convergence to equilibrium, if $x<x^{*}, F(x)$ must be greater than zero; if $x>x^{*}, F(x)$ must be smaller than zero.

If $F(x)=0$, the equilibrium of the replicator equation of $\mathrm{A}$ can be described as:

$$
\begin{gathered}
x^{*}=0 ; x^{*}=1 ; \\
y^{*}=\left(C_{a}+\gamma D_{a}\right) /\left(\mu D_{b} P_{a}+\alpha D_{a}-D_{b} P_{a}+\beta D_{b}\right)
\end{gathered}
$$

Case 1: Under $C_{a}+\gamma D_{a}>\mu D_{b} P_{a}+\alpha D_{a}-D_{b} P_{a}+\beta D_{b}$, for any $y(0 \leq y \leq 1)$, if there exists $F^{\prime}\left(x^{*}=0\right)<0$, then $x^{*}=0$ is the evolutionary equilibrium strategy of A.

Case 2: Under $C_{a}+\gamma D_{a}<\mu D_{b} P_{a}+\alpha D_{a}-D_{b} P_{a}+\beta D_{b}$, for any $y>y^{*}$, there exists $F^{\prime}\left(x^{*}=0\right)<0$, then $x^{*}=0$ is the evolutionary equilibrium strategy of A.

Similarly, if $F(x)=0$, the equilibrium of the replicator equation of $\mathrm{B}$ can be described as:

$$
\begin{gathered}
y^{*}=0 ; y^{*}=1 ; \\
x^{*}=\left(C_{b}+\gamma D_{b}\right) /\left(\mu D_{a} P_{b}+\alpha D_{b}-D_{a} P_{b}+\beta D_{a}\right)
\end{gathered}
$$

Case 3: Under $C_{b}+\gamma D_{b}>\mu D_{a} P_{b}+\alpha D_{b}-D_{a} P_{b}+\beta D_{a}$, for any $x(0 \leq x \leq 1)$, if there exists $F^{\prime}\left(y^{*}=0\right)<0$, then $y^{*}=0$ is the evolutionary equilibrium strategy of $\mathrm{B}$.

Case 4: Under $C_{b}+\gamma D_{b}<\mu D_{a} P_{b}+\alpha D_{b}-D_{a} P_{b}+\beta D_{a}$, for any $x>x^{*}$, if there exists $F^{\prime}\left(y^{*}=1\right)<0$, then $y^{*}=1$ is the evolutionary equilibrium strategy of $\mathrm{B}$; for any $x<x^{*}$, if there exists $F^{\prime}\left(y^{*}=0\right)<0$, then $y^{*}=0$ is the evolutionary equilibrium strategy of B.

\subsection{Dynamic evolution simulation}

In the preceding subsection, the evolutionary equilibrium strategies of A and B were analyzed separately. In actual evolutionary games, however, the situation of both sides should be considered, and the evolutionary equilibrium strategy could only be generated through the interaction between the two sides. The interaction produces four results, because of the different parameters and situations of the two parties.

Results 1: If $A$ is in Case 1 and $B$ is in Case 3 (Figure 1), the two sides have bounded rationality. The initial strategies chosen by A and B are not necessarily optimal. Through repeated game, it is found that the non-IS is better than the IS. After several stages of learning and adjustment, the two sides converged to $(0,0)$. Hence, the evolutionary equilibrium strategy for A and B is \{non-IS, non-IS \} in this situation. 


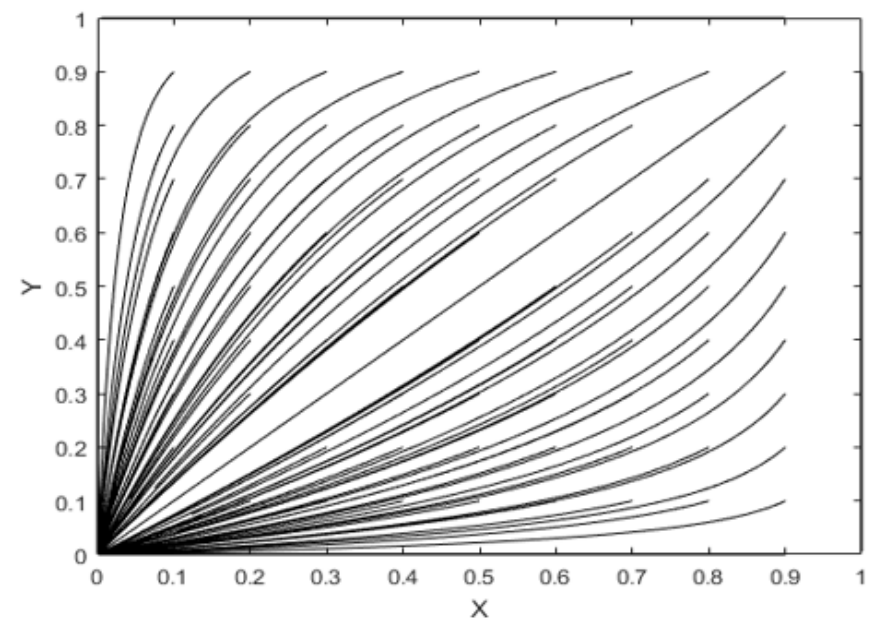

Figure 1. Dynamic evolution of A in Case 1 and B in Case 3

Results 2: If A is in Case 1 and B is in Case 4 (Figure 2), the two sides eventually converged to $(0,0)$. The evolutionary equilibrium strategy for $\mathrm{A}$ and $\mathrm{B}$ is still \{non-IS, non-IS in this situation.

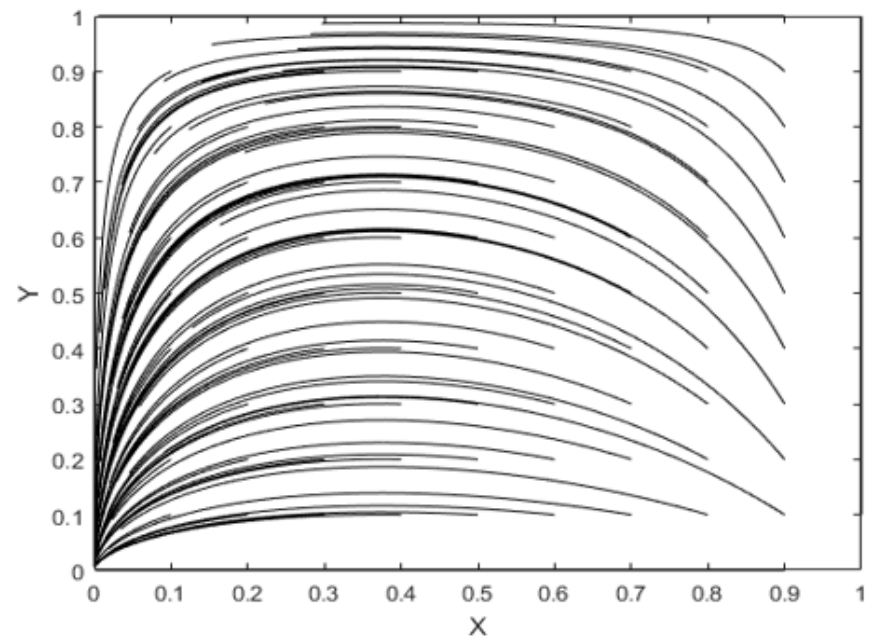

Figure 2. Dynamic evolution of $A$ in Case 1 and B in Case 4

Results 3: If A is in Case 2 and B is in Case 3 (Figure 3), the two sides eventually converged to $(0,0)$. The evolutionary equilibrium strategy for A and B is still \{non-IS, non-IS \} in this situation.

Result 4: If A is in Case 2 and B is in Case 4 (Figure 4), the evolutionary equilibrium strategies of $\mathrm{A}$ and $\mathrm{B}$ depends on the value of the saddle point $F\left(x^{*}, y^{*}\right)$. With CFE as the dividing line, if the saddle point falls in the region OCEF, the two sides will converge to point $\mathrm{O}(0,0)$, i.e. the evolutionary equilibrium strategy for $\mathrm{A}$ and $\mathrm{B}$ is $\{$ non-IS, non-IS $\}$; if the saddle point falls in the region CFED, the two sides will converge to point $\mathrm{D}(1,1)$, i.e. the evolutionary equilibrium strategy for A and B is $\{$ IS, IS $\}$.

The following conclusions can be drawn from the above dynamic evolution simulation:

(1) According to Results 1,2 and 3, if inequalities $C_{a}+$ $\gamma D_{a}>\mu D_{b} P_{a}+\alpha D_{a}-D_{b} P_{a}+\beta D_{b} \quad$ and $\quad C_{b}+\gamma D_{b}>$ $\mu D_{a} P_{b}+\alpha D_{b}-D_{a} P_{b}+\beta D_{a}$ hold, then either $D_{b} P_{a}-\beta D_{b}>$ $\mu D_{b} P_{a}+\alpha D_{a}-C_{a}-\gamma D_{a}$ or $D_{a} P_{b}-\beta D_{a}>\mu D_{a} P_{b}+\alpha D_{b}-$ $C_{b}-\gamma D_{b}$ is true.

(2) According to Result 4, only if inequalities $C_{a}+\gamma D_{a}>$ $\mu D_{b} P_{a}+\alpha D_{a}-D_{b} P_{a}+\beta D_{b}$ and $C_{b}+\gamma D_{b}>\mu D_{a} P_{b}+\alpha D_{b}-$
$D_{a} P_{b}+\beta D_{a}$ hold, both $D_{b} P_{a}-\beta D_{b}>\mu D_{b} P_{a}+\alpha D_{a}-C_{a}-$ $\gamma D_{a}$ and $D_{a} P_{b}-\beta D_{a}>\mu D_{a} P_{b}+\alpha D_{b}-C_{b}-\gamma D_{b}$ are true. In this case, $\mathrm{A}$ and $\mathrm{B}$ are likely to adopt the evolutionary equilibrium strategy $\{$ IS, IS $\}$. The probability for both sides to take this strategy changes with the saddle point $F\left(x^{*}, y^{*}\right)$. The smaller the value of saddle point, the larger the area of CFED, and the greater the probability of $\{$ IS, IS $\}$. The inverse is also true.

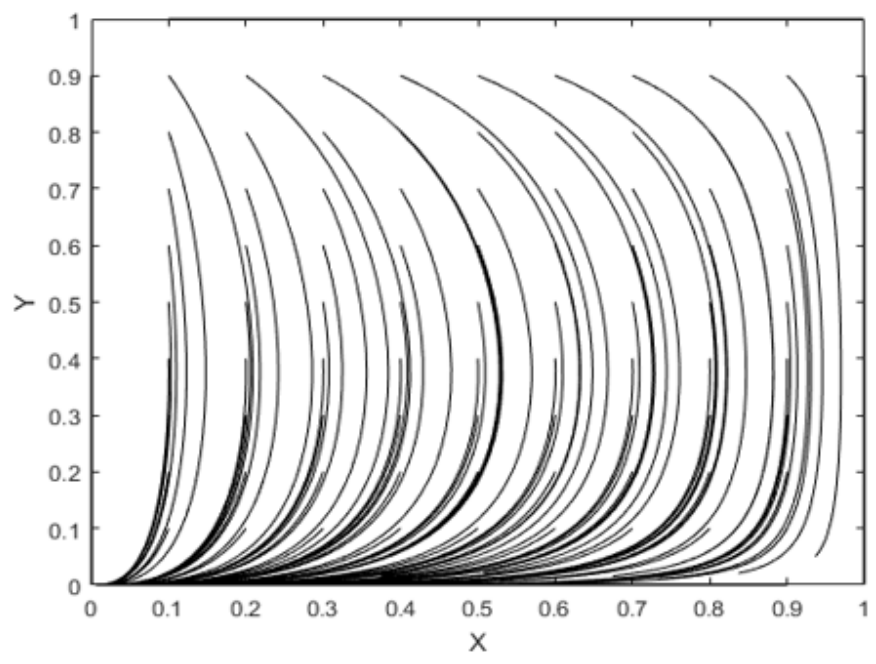

Figure 3. Dynamic evolution of $\mathrm{A}$ in Case 2 and $\mathrm{B}$ in Case 3

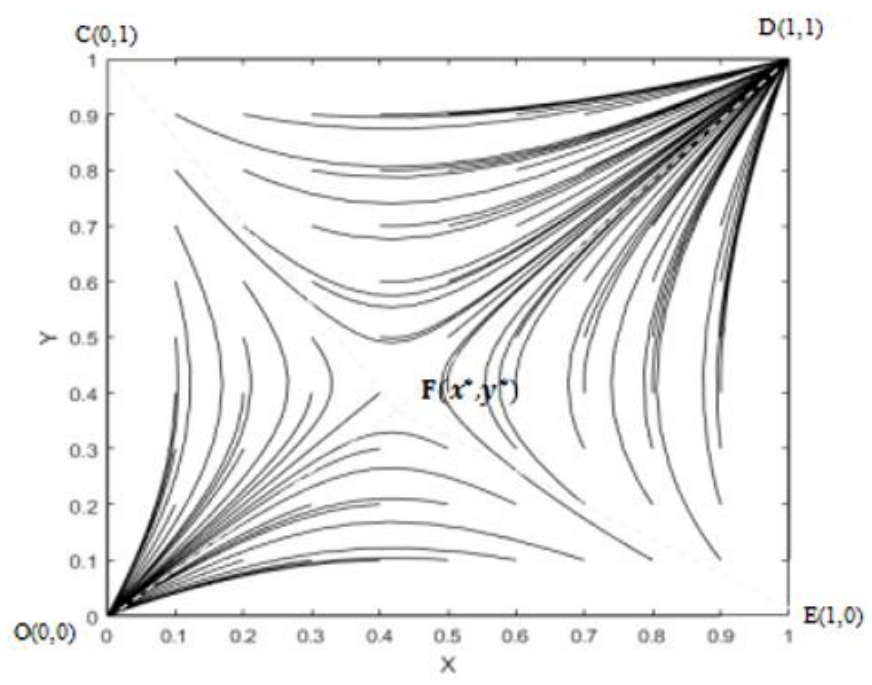

Figure 4. Dynamic evolution of A in Case 2 and B in Case 4

\subsection{Parameter analysis}

The above analysis shows that the final evolutionary equilibrium strategy of FPSC enterprises depends on the value of saddle point $F\left(x^{*}, \mathrm{y}^{*}\right)$ in game model:

$$
\begin{aligned}
& x *=\left(\mathrm{C}_{\mathrm{b}}+\gamma \mathrm{D}_{\mathrm{b}}\right) /\left(\mu \mathrm{D}_{a} \mathrm{P}_{\mathrm{b}}+\alpha \mathrm{D}_{\mathrm{b}}-\mathrm{D}_{a} \mathrm{P}_{\mathrm{b}}+\beta \mathrm{D}_{\mathrm{a}},\right. \\
& y *=\left(\mathrm{C}_{\mathrm{a}}+\gamma \mathrm{D}_{\mathrm{a}}\right) /\left(\mu \mathrm{D}_{b} \mathrm{P}_{\mathrm{a}}+\alpha \mathrm{D}_{\mathrm{a}}-\mathrm{D}_{b} \mathrm{P}_{\mathrm{a}}+\beta \mathrm{D}_{\mathrm{b}}\right) .
\end{aligned}
$$

In the FPSC, the saddle point value is mainly affected by the IS degree, the information absorption capacity, the IS cost, the incentive coefficient, penalty coefficient, risk coefficient and synergy coefficient. The impact of each influencing factor on the IS between FPSC enterprises is detailed below:

(1) With the growing IS degree $D_{i}$, both the numerator and denominator of $x^{*}$ and $y^{*}$ increases. Then, the saddle point $\mathrm{F}$ will move to the lower left of the dynamic evolution map, and the CFED area will expand. In this way, the enterprises will be 
more likely to choose the IS strategy.

(2) With the growth in information absorption capacity $p_{i}$, incentive coefficient $\alpha$, penalty coefficient $\beta$, or synergy coefficient $\mu$, the denominators of $x^{*}$ and $y^{*}$ will increase. Then, the saddle point $\mathrm{F}$ will move to the lower left of the dynamic evolution map, and the CFED area will expand. In this way, the enterprises will be more likely to choose the IS strategy.

(3) With the growth in the IS cost $c_{i}$, or risk coefficient $\gamma$, the numerators of $x^{*}$ and $y^{*}$ will increase. Then, the saddle point $\mathrm{F}$ will move to the upper right of the dynamic evolution map, and the CFED area will shrink. In this way, the enterprises will be less likely to choose the IS strategy.

\section{COUNTERMEASURES}

\subsection{Raising the IS awareness}

It is necessary to cultivate and enhance the IS awareness of FPSC enterprises. The basis of the IS lies in the mutual trust between the enterprises, which in turn depends on the effectiveness of communication. FPSC enterprises should pursue more cultural exchanges through cooperation and communication, rather than blindly pursue their own interests through the traditional model of competition. The effective communication between FPSC enterprises helps to clarify the information demand of each side, enabling each side to provide and receive the pertinent information quickly and efficiently at a low cost. In this way, FPSC enterprises will achieve a win-win and receive mutual benefits. The key point of raising the IS awareness lies in improving the information literacy of forest farmers.

\subsection{Enhancing information absorption capacity}

In the age of big data, information explosion is the common problem for all FPSC enterprises. To gain a competitive edge, each enterprise faces the urgent task to select and utilize information out of the massive data. Firstly, the information management system should be improved across-the-board, and a pool of talents in information technology must be trained, providing a guarantee for information operations. Secondly, FPSC enterprises should improve the information literacy of their employees (e.g. encourage autonomous learning of information technology and screen out those with poor information literacy), such that information can flow smoothly inside the organizational structure. Thirdly, FPSC enterprises should build a corporate culture that advocates open sharing, which is conducive to internal communication between employees, as well as the partnership and trust between enterprises.

\subsection{Creating an IS mechanism}

In the FPSC, each enterprise acts independently, and select its IS strategy on a voluntary basis. The IS between FPSC enterprises calls for a mature IS mechanism. Firstly, incentive measures should be introduced to increase the attractiveness of the IS. For example, the enterprises that actively implement the IS can be rewarded with high-quality information and targeted brand publicity. Secondly, penalty measures should be adopted to punish the enterprises that adopt speculative behavior, such as imposing fines on them or adding them to a blacklist. Thirdly, risk prevention measures should be taken to enhance the information security between enterprises. For instance, FPSC enterprises can sign agreements to maintain the trust and cooperation, and set access rights to the IS platform.

\subsection{Strengthening IS coordination}

The IS between enterprises is fundamental to the dynamic cooperation in the FPSC. The IS between the enterprises must be coordinated well, making the cooperative relationship longlasting. Therefore, the IS coordination should be strengthened by defining a common goal for all enterprises through consultation. Then, each enterprise could strive to achieve the common goal, while realizing its individual goals. Meanwhile, FPSC enterprises should maintain effective communication, and clearly understand the information demand of each party, making the IS more targeted. If any dispute arises in the course of the IS, the two sides should work together to resolve the dispute through communication, and minimize its impact on the cooperative relationship. In addition, the enterprises should provide timely feedbacks on the IS, such that the sharing process can be optimized according to the specific situation.

\section{CONCLUSIONS}

Drawing on evolutionary game theory, this paper establishes a model for the dynamic decision-making of the IS between FPSC enterprises. According to the relevant literature several influencing factors of the IS were identified, including, the IS degree, information absorption capacity, the IS cost, incentive coefficient, penalty coefficient, risk coefficient and synergy coefficient. Through Matlab simulation, the impact of each factor on the IS decisions of each side was discussed based on our model. The results show that the probability for each side to choose the IS is positively affected by the IS degree, information absorption capacity, incentive coefficient, penalty coefficient and synergy coefficient, and negatively affected by the IS cost and risk coefficient. Finally, several countermeasures were presented to promote the IS between FPSC enterprises, namely, raising the IS awareness, enhancing information absorption capacity, creating an IS mechanism, and strengthening IS coordination. The future research will examine even more influencing factors of the IS between FPSC enterprises, and apply our findings in the analysis of specific cases.

\section{REFERENCES}

[1] Lee, H.L., So, K.C., Tang, C.S. (2000). The value of information sharing in a two-level supply chain. Management Science, 46(5): 626-643. http://dx.doi.org/10.1287/mnsc.46.5.626.12047

[2] Thonemann, U.W. (2002). Improving supply-chain performance by sharing advance demand information. European Journal of Operational Research, 142(1): 81107. http://dx.doi.org/10.1016/S0377-2217(01)00281-8

[3] Dai, H., Li, J., Yan, N., Zhou, W. (2016). Bullwhip effect and supply chain costs with low-and high-quality information on inventory shrinkage. European Journal of Operational Research, 250(2): 457-469. https://doi.org/10.1016/j.ejor.2015.11.004

[4] Mishra, B.K., Raghunathan, S., Yue, X. (2007). 
Information sharing in supply chains: Incentives for information distortion. IIE Transactions, 39(9): 863-877. http://dx.doi.org/10.1080/07408170601019460

[5] Ye, F., Chen, X.M., Lin, Q. (2012). Analysis of supply chain's demand information sharing values based on decision-maker's risk aversion. Journal of Industrial Engineering/ Engineering Management, 26(3): 176-183. https://doi.org/10.3969/j.issn.1004-6062.2012.03.025

[6] Masuchun, W., Davis, S., Rangsaritratsamee, R. (2006). System for supply network management. Information Technology and Management, 7(4): 315-333. https://doi.org/10.1007/s10799-006-0279-5

[7] Bai, S.Z., Wang, X., Zhang, S.T. (2010). Research on information sharing incentive and supervisory mechanism in supply chain. In 2010 International Conference on Logistics Systems and Intelligent Management (ICLSIM), 2: 641-645. https://doi.org/10.1109/ICLSIM.2010.5461342

[8] Teunter, R.H., Babai, M.Z., Bokhorst, J.A., Syntetos, A.A. (2018). Revisiting the value of information sharing in two-stage supply chains. European Journal of Operational Research, 270(3): 1044-1052. http://dx.doi.org/10.1016/j.ejor.2018.04.040

[9] Zhao, J., Zhu, H., Zheng, S. (2018). What is the value of an online retailer sharing demand forecast information? Soft Computing, 22(16): 5419-5428. http://dx.doi.org/10.1007/s00500-018-3091-3

[10] Babai, M.Z., Boylan, J.E., Syntetos, A.A., Ali, M.M. (2016). Reduction of the value of information sharing as demand becomes strongly auto-correlated. International Journal of Production Economics, 181: 130-135. http://dx.doi.org/10.1016/j.ijpe.2015.05.005

[11] Sabitha, D., Rajendran, C., Kalpakam, S., Ziegler, H. (2016). The value of information sharing in a serial supply chain with AR (1) demand and non-zero replenishment lead times. European Journal of Operational Research, 255(3): 758-777. http://dx.doi.org/10.1016/j.ejor.2016.05.016

[12] Rached, M., Bahroun, Z., Campagne, J.P. (2015). Assessing the value of information sharing and its impact on the performance of the various partners in supply chains. Computers \& Industrial Engineering, 88: 237253. http://dx.doi.org/10.1016/j.cie.2015.07.007

[13] Scott, A. (2015). The value of information sharing for truckload shippers. Transportation Research Part E: Logistics and Transportation Review, 81: 203-214. http://dx.doi.org/10.1016/j.tre.2015.07.002

[14] Choudhary, D., Shankar, R. (2015). The value of VMI beyond information sharing in a single supplier multiple retailers supply chain under a non-stationary $(\mathrm{Rn}, \mathrm{Sn})$ policy. Omega, 51: 59-70. http://dx.doi.org/10.1016/j.omega.2014.09.004

[15] Feldmann, M., Müller, S. (2003). An incentive scheme for true information providing in supply chains. Omega, 31(2): $\quad$ 63-73. $\quad \mathrm{http}: / / \mathrm{dx}$.doi.org/10.1016/S03050483(02)00096-8

[16] Fan, R.G., Dong, L.L. (2018). The dynamic analysis and simulation of government subsidy strategies in lowcarbon diffusion considering the behavior of heterogeneous agents. Energy Policy, 117: 252-262. https://doi.org/10.1016/j.enpol.2017.12.003

[17] Zhu, G., Pan, G., Zhang, W. (2018). Evolutionary game theoretic analysis of low carbon investment in supply chains under governmental subsidies. International Journal of Environmental Research and Public Health, 15(11): 2465-2491. https://doi.org/10.3390/ijerph15112465

[18] Zhang, C. (2016). Small and medium-sized enterprises closed-loop supply chain finance risk based on evolutionary game theory and system dynamics. Journal of Shanghai Jiaotong University (Science), 21(3): 355364. https://doi.org/10.1007/s12204-016-1733-0

[19] Fang, D.B., Zhao, C.Y., Yu, Q. (2018). Government regulation of renewable energy generation and transmission in China's electricity market. Renewable and Sustainable Energy Reviews, 93: 775-793. https://doi.org/10.1016/j.rser.2018.05.039

[20] Zhang, H.M., Xu, Z.D., Zhou, D.Q., Qiu, Y.M., Shen, D.Y. (2018). Targeted poverty alleviation using photovoltaic power in China: Identifying financial options through a dynamic game analysis. Resources, Conservation and Recycling, 139: 333-337. https://doi.org/10.1016/j.resconrec.2018.09.006

[21] Zhao, X.G., Ren, L.Z., Zhang, Y.Z., Wan, G. (2018). Evolutionary game analysis on the behavior strategies of power producers in renewable portfolio standard. Energy, 162:

505-516. https://doi.org/10.1016/j.energy.2018.07.209

[22] Qi, Y.Z., Liu, Y.L., Wu, Q.W. (2017). Non-cooperative regulation coordination based on game theory for wind farm clusters during ramping events. Energy, 132: 136146. https://doi.org/10.1016/j.energy.2017.05.060

[23] Zhang, M., Li, H. (2018). New evolutionary game model of the regional governance of haze pollution in China. Applied Mathematical Modelling, 63: 577-590. https://doi.org/10.1016/j.apm.2018.07.008 